\title{
Predictors of Psychological Tension Among Refugees Living in Montréal
}

\author{
André Jacob, Joseph J. Lévy, Louis Robert Frigault and Jocelyne Bertot
}

Québec, like the rest of Canada and other industrialized countries, is experiencing a growing influx of refugees from various regions around the world. In 1991, nearly 30 percent of all immigrants were refugees from Africa, Latin America, Asia, Europe or the Middle East. For refugees, integration inevitably raises complex economic, sociocultural and psychological problems. For these populations, settlement in the host country is often accompanied by multiple tensions and stresses, leading to a profound disorganization of personal and social identity for refugees and increasing their risk of psychological imbalance.

Refugees may experience a range of diverse psychological problems such as detachment, anxiety, irritability, emotional instability, aggression and depression (Beiser 1990; Tousignant 1992). These problems have been linked to numerous factors. For some authors, pre-migratory factors (conditions surrounding the departure from the country of origin), traumatic experiences (war, torture, rape, etc.) and the time spent in transit play important roles in the modulation of mental health (Beiser, Turner and Gamesan 1989). For others, mental health depends on the conditions of integration in the host country (Murphy 1977). Economic and sociocultural integration, as well as the social resources related to family and to communal support, also seem to play important roles in maintaining mental health. Finally, the ability to communicate in the language of the country is one of the

The authors are researchers at the Centre de Recherches surles relations interethniques et le racisme, Université du Québec a Montréal. This research has been made possible by a grant from the Ministry of Health and Welfare, Ottawa, Canada. most important variables determining psychological adaptation (Lambert and Taylor 1990).

Women often suffer more intensely from psychological problems associated with their status in the country of origin, amplified by the consequences of exile. Their level of education and professional skills (which are often lower than those of men-depending on the country of origin), social isolation, and childcare responsibilities make their adaptation and integration into the host country more difficult (Camus-Jacques 1989).

These studies show the complex interplay of factors which determine the mental health of refugees. In order to better understand them, we will present here the preliminary results of a study on the postmigratory predictors of psychological tensions among five different groups of refugees living in Montréal. This comparative research will illustrate the common underlying variables affecting the development of psychological wellbeing among refugees.

\section{Methodology}

\section{The Subjects}

Within the framework of a comparative analysis of the integration of refugees and the use of social and health services, 472 respondents (119 Cambodians, 97 Bulgarians, 92 Guatemalans, 93 Tamils and 71 Ethiopians; 245 women and 227 men) completed, between September 1992 and April 1993, a questionnaire composed mainly of closed questions pertaining to different dimensions of their economic, sociocultural and psychological integration. The immigration status of the respondents varied; while almost all entered Canada as refugees, 62.4 percent had become permanent residents,
8.5 percent had attained refugee status, 10 percent were awaiting status determination and 18.9 percent were Canadian citizens. The mean age of the respondents was 32.9 years. The majority, 53 percent, were married or lived in cohabitation, 34.7 percent were single and 12 percent were separated, divorced or widowed. On average, they had been living in Montréal for three years and five months.

\section{The Questionnaire}

The questionnaire was translated from French to English and to the different languages of the respondents. Responses were later retranslated into French in order to ensure comparability. Questionnaires were completed by the respondents under the supervision of research assistants and interpreters.

The questionnaire included questions about the refugees' profile and integration. The level of psychological tension was established from a global scale (ranging from 4 to 28 ) which included the following dimensions: worried, discouraged, nervous, anxious, tired, guilty and angry in the last six months. They were evaluated on a four point Likert scale (from not at all, to very much). The Cronbach's alpha was 0.75 .

The independent variables were as follows: length of stay in Québec; level of education; knowledge of French and English (measured by a global scale evaluating their speaking, reading and writing abilities in each of these languages); family income; employment; difficulties in adaptation in different areas (pace of lifestyle, climate, diet, work and housing, family and community relations variables included in a single global scale); social support (size of household, ethnic origin of friends, discussion of personal problems, appreciation of neighbours and

Refuge, Vol. 13, No. 9 (February 1994) 
expected help from them); and scale of participation in intra-and extra-communal activities. Several variables dealt with different perceptions of integration into Québec society: progression in material wellbeing; adaptation to social environment and lifestyle; evaluation of professional prospects in Québec; level of satisfaction concerning social relations and perception of the level of welcoming by the Québecois; and desire to return to their native country. Age, civil status, sex as well as immigration status (refugee, resident, citizen, etc.) were also included as variables.
Analysis of variance (ANOVA) and $t$-tests were carried out in order to evaluate the effect of each of the independent variables on the score of psychological distress. Following this first analysis, a logistic regression analysis was carried out in order to determine the most important predictors.

\section{Results}

As shown in Table 1, the level of psychological tension, which was relatively low, varied significantly according to the national origin of the respondents. The post hoc analysis demonstrated that the Cambodians experienced the lowest levels of distress, differing significantly from all other groups, except the Guatemalans. The Tamils, however, had the highest levels of distress, differing significantly from all other groups, except the Bulgarians, who differed significantly from the Guatemalans.

Several postmigratory factors appear to have modulated the psychological scores. Refugees living in Québec for less than one year had higher levels of distress than those who had been in Quebec between one and three years. Citizenship status also modulated the levels of tension; those

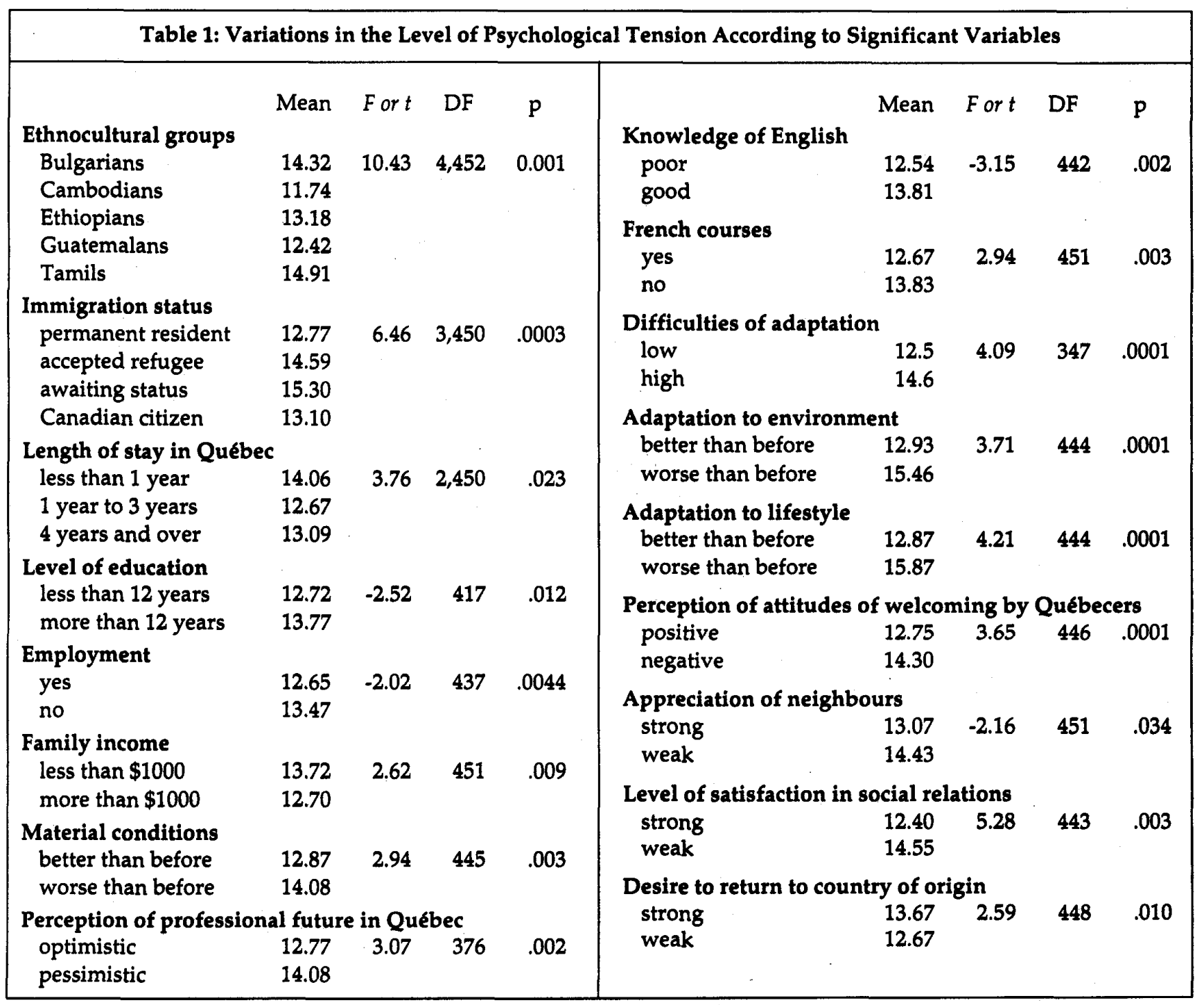


who had permanent resident status in Canada reported less psychological tension than those who had been accepted as refugees or were awaiting refugee status. Canadian citizens were also less tense than those awaiting status determination.

Respondents with higher levels of education, as well as those with a good knowledge of English or those who did not attend French language courses were more likely to report higher levels of psychological tension. Refugees who were unemployed or had a low family income, as well as those experiencing difficulties adapting to the Québec social environment, reported higher levels of tension. In contrast, an optimistic evaluation of a professional future in Québec resulted in lower levels of psychological tension.

Positive perceptions concerning improved material conditions, adaptation to the social environment and lifestyle of Québec were also indicators of lower tension levels. The same effect was present when respondents reported positively about perceived Québecois hospitality, social relations, and a weak desire to return to their native country.

Other factors such as household size, willingness to discuss personal problems, perceived willingness of their neighbours to assist, knowledge of French, the ethnic origin of friends, participation in intra-or extra-communal activities, age, civil status, and sex did not significantly vary with the level of psychological distress.

\section{Discussion}

This comparative study of refugees living in Montréal provided support for the presence of important variables in the modulation of psychological distress within the five ethnocultural groups considered. In this respect, two groups, the Bulgarians and the Tamils could be clearly distinguished from the others, and this was confirmed by logistic regression. Table 2 indicates that being a member of either of these two groups was associated with high levels of psychological tension. This

Table 2. Odd Ratios for Predictors of Psychological Tension According to Logistic Regression

$\begin{array}{lrr}\text { Variables } & \text { Odd Ratio } & \text { CI (95\%) } \\ \text { Bulgarians } & 3.79 & (2.12 ; 6.77) \\ \text { Tamils } & 2.91 & (1.62 ; 5.23) \\ \text { Amelioration of material conditions } & 0.68 & (0.48 ; 0.95) \\ \text { Desire to return to country of origin } & 0.75 & (0.59 ; 0.93) \\ \text { Perception of professional } & & \\ \quad \text { future in Québec } & 0.80 & (0.64 ; 0.99) \\ \quad \text { Family income } & 0.85 & (0.73 ; 0.99) \\ \text { Difficulties of adaptation } & 0.87 & (0.78 ; 0.97)\end{array}$

Note: Final Chi-square model: Chi-square 69.30; df 7; $p<0.0001$.

could be due to specific factors linked to their integration in Québec society.

Preliminary results of our research (Jacob, Lévy, Bertot et al. 1993) showed that both groups were confronted with problems which differentiated them from other groups included in the study. The highest percentage of persons awaiting refugee status was found among Bulgarians and this finding can explain in part why they experienced higher levels of psychological tension. Secondly, the Bulgarians reported the highest level of university education-a finding which could be a second source of tension, since, in spite of this asset and the fact that many speak fluent French, they had the highest level of unemployment among the study groups, as well as the greatest drop in living standards, compared to the other groups.

While economic factors seemed prominent in explaining the higher levels of tension experienced by the Bulgarians, another configuration could be pinpointed for the Tamils. Although theireconomicstanding was slightly higher than the Bulgarians, they had more difficulties fitting into the sociocultural milieu of Montreal, reporting high levels of stress in adapting to their new environment, as well as the lowest level of fluency in French, the lowest number of French-Canadian friends and the most negative perception of the hospitality of the
Québec population. They also reported the greatest difficulty in adapting to the social environment or lifestyle. Furthermore, the Tamils were most likely to report a desire to return to their native country. All these dimensions seem to indicate a less satisfactory integration in Montréal than in Toronto (Shier and Coomarasamy 1988; Mills 1993).

As we shall now see, several factors affected the level of psychological distress for the study population as a whole. Length of stay in Québec had a significant impact, with the first year being crucial in this respect. This effect has also been reported in other studies on refugees from Southeast Asia (Beiser et al. 1989). The high initial stress levels can be linked to the level of stress experienced during the premigratory context. However, one should note that stress levels increased slightly after three years. This could be due to new stresses related to problems arising from the integration process. Awaiting the determination of refugee status could also have contributed to tensions, since the lengthy judicial process, and the unpredictability of the outcome were certainly important sources of stress. However, this factor was not found to be a significant determinant of psychological wellbeing.

Higher levels of education were associated with higher levels of psy- 
chological tension. The underemployment generally experienced by immigrants and refugees (Tousignant 1992) can have a deeper effect on the psychological wellbeing of the more educated, since they would suffer more from professional changes.

Several economic factors were linked to the psychological score: employment, family income (even if it was lower than in the general population), perceptions of progress in the level of economic standing, and an optimistic perspective concerning one's professional future were all sig- integration into the French milieu, especially among groups, like the Tamils, for whom English is one of their mother tongues. Conversely, learning French through immersion courses would enhance integration by increasing possibilities of communication with the host population (Beiser 1990). But, as shown by the logistic regression, these factors did not predict psychological wellbeing among refugees.

While satisfactory social relations and positive perceptions concerning adaptation to the Québecois social en-

\section{While many variables intervened in its modulation, logistic regression suggested that psychological tension was predomi- nantly modulated by socioeconomic factors and stressors linked to the acculturation process as well as nostalgia for the country of origin. These results indicated that the psychological tension of refugees was dependent on objective indicators as well as sociopsychological dimensions.}

nificant factors in modulating psychological wellbeing among the refugees interviewed. The logistic analysis (Table 2) showed that the last three factors were significant predictors, confirming the crucial role that economic integration plays in the development of the mental health of the refugees (Beiser 1990). In this respect, it seems that objective economic conditions alone do not positively influence psychological health. In addition, cognitive factors linked to the evaluation of economic progress also have an effect by empowering refugees in their quest toward a brighter future.

Several variables which measure sociocultural integration affected the scale, but their impact disappeared almost completely when they were entered in the logistic analysis. Some linguistic variables (knowledge of English, participation in French courses) did affect the psychological score, however, the linguistic situation in Québec may explain the paradoxical results found in this research. Since English is not the predominant language in Québec, speaking fluent English could be an impediment to vironment were strong positive predictors of psychological wellbeing, difficulties encountered in the adaptation process were one of the main negative predictors revealed by logistic regression (Table 2), suggesting that stresses and conflicts linked to acculturation are, as proposed by Berry (1990), a source of reduced mental health status of refugees. Finally, it seems that a strong desire to return to one's native country is also a predictor of psychological distress, indicating that for some refugees, life in their new country cannot erase the memories and nostalgia for their native country. As reported by Beiser et al. (1989), nostalgic people have a tendency to be more depressed than those who are oriented toward the present and the future. This was also the case in our study.

\section{Conclusion}

The results of this study of refugees living in Montréal led us to conclude that the level of psychological tension varied according to the ethnic group studied and was higher for Bulgarians and Tamils. While many variables intervened in its modulation, logistic re- gression suggested that psychological tension was predominantly modulated by socioeconomic factors and stressors linked to the acculturation process as well as nostalgia for the country of origin. These results indicated that the psychological tension of refugees was dependent on objective indicators as well as sociopsychological dimensions. Both have to be taken into account when defining programs of intervention among these populations in order to increase their wellbeing and enable them to successfully integrate into their new physical and sociocultural environment.

\section{References}

Beiser, Morton. 1990. "Mental Health of Refugees in Resettlement Countries." In Holtzman, Wayne $\mathrm{H}$. and Bornemann, Thomas H. (eds.) Mental Health of Immigrants and Refugees. Austin: Hogg Foundation for Mental Health.

Beiser, M., Turner, R. and S. Gamesan. 1989. "The Mental Health of Immigrants and Refugees in Canada." In Santé, Culture, Health 5:197-213.

Berry, John W. 1990. "Acculturation and Adaptation: A General Framework." In Holtzman, Wayne $H$. and Bornemann, Thomas H. (eds.) Mental Health of Immigrants and Refugees. Austin: Hogg Foundation for Mental Health.

Camus-Jacques, Geneviéve. 1989. "Refugee Women: The Forgotten Majority." In Loescher, Gil and Monahan, Laila (eds.) Refugees and International Relations. Oxford Clarendon Press.

Jacob, A., Lévy, J., Bertot, J., Sauve, S., Poblete, H. and Frigault, L.R. 1993. Integration de Quelques Groupes de REfugiés d Montréal. Rapport de Recherche Département de Travail Social.

Lambert, W. and Taylor, D. M. 1990. "Language and Culture in the Lives of Immigrants and Refugees." In Holtzman, Wayne H. and Bornemann, Thomas H. (eds.) Mental Health of Immigrants and Refugees. Austin: Hogg Foundation for Mental Health.

Mills, Megan Stuart. 1993. "Mental Health Resilience of Refugees: The case of Tamil refugees." In Refuge 13, 3:28-29.

Murphy, H.B.M. 1977. "Migration, Culture and Mental Health." In Psychological Medicine 7:677-684.

Shire, Safia and Coomarasamy, Sudha. 1988. Somalian and Tamil Refugee Women in Montreal. Twenty case studies. Rapport d'une Recherche Effectuée au Tyndale St. Georges Community Center. Montréal.

Tousignant, Michel. 1992. Les Origines Sociales et Culturelles des Troubles Psychologiques. Paris: Presses Universitaires de France. $\square$ 